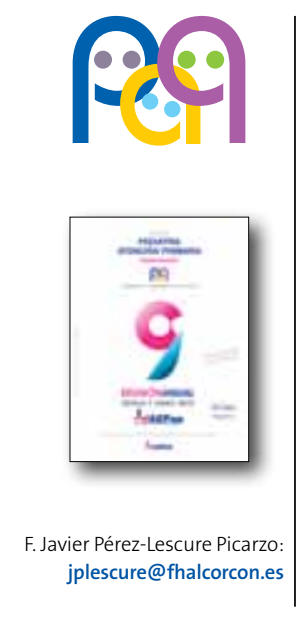

\title{
El niño deportista: evaluación cardiológica en Atención Primaria
}

\section{F. J. Pérez-Lescure Picarzo}

Cardiología Infantil. Hospital Universitario Fundación Alcorcón. Alcorcón, Madrid. España.

\section{OBJETIVOS DEL TALLER}

- Conocer cómo se realiza una evaluación predeportiva en un niño para descartar patología cardiovascular y especialmente patología con riesgo de muerte súbita.

- Recordar los antecedentes familiares, personales y los hallazgos de la exploración física que pueden estar relacionados con patología cardiaca potencialmente grave y precisar una derivación del paciente para un estudio más exhaustivo.

- Interpretar los hallazgos electrocardiográficos que pueden hacernos sospechar patología cardiovascular de riesgo para el deporte.

- Cómo valorar los síntomas durante el ejercicio en los niños.

\section{INTRODUCCIÓN}

Se define muerte súbita cardiaca (MSC) como la muerte que ocurre durante la hora siguiente al inicio de los síntomas en ausencia de anomalías cardiovasculares conocidas, excluyendo fallecimientos relacionados con causas respiratoria, cerebrovascular o drogas ${ }^{1}$. La incidencia de MSC en menores de 35 años oscila entre 0,3 y 3,6/100 000 personas/año ${ }^{2}$ con claro predominio en varones (relación 5-10:1)3. El 90\% de las muertes súbitas son de origen cardiovascular 4 .

Las principales causas de MSC en deportistas jóvenes (<35 años) son miocardiopatías (hipertrófica, arritmogénica del ventrículo derecho, dilatada) y anomalías congénitas de las arterias coronarias ${ }^{5}$. Otras menos frecuentes son canalopatías (síndrome de Brugada, síndrome de QT largo, síndrome de OT corto), síndromes de preexcitación (tipo WolfParkinson-White), valvulopatías (estenosis aórtica, prolapso mitral), disección/ruptura aórtica en el contexto de síndrome de Marfan (SM), miocarditis y commotio cordis ${ }^{6}$.

Existe consenso general en la necesidad de realizar un cribado predeportivo, aunque con enormes divergencias en cuanto a los protocolos y a las bases legales en distintos países. Las principales guías han sido elaboradas por la American Heart Association $(\mathrm{AHA})^{7}$ y la Sociedad Europea de Cardiología $(E S C)^{8}$. Ambas incluyen cuestionarios más o menos exhaustivos en cuanto a anamnesis, antecedentes personales y familiares, además de una minuciosa exploración física. Las controversias se dan en la realización o no de un electrocardiograma (ECG) de forma sistemática, y en el personal encargado de realizar el reconocimiento.

La mayoría de autores ${ }^{9}$ recomiendan incluir el ECG en la sistemática de la evaluación predeportiva, mientras que la AHA argumenta su baja especifici- 
dad, alto coste económico y la dificultad de implicar a los médicos en la realización de reconocimientos médicos de despistaje cardiovascular.

En España, disponemos de un protocolo de reconocimiento cardiológico previo a la práctica deportiva elaborado por la Sociedad Española de Cardiología (SEC) en el año $2000^{10}$. Aunque puede ser aplicado a niños, no fue diseñado específicamente para este grupo de edad, y habitualmente no se realiza en el ámbito de las revisiones predeportivas pediátricas en nuestro país.

\section{VALORACIÓN PREDEPORTIVA Y/O DE UN NIÑO CON SÍNTOMAS AL EJERCICIO (ORIENTADO A DESCARTAR PATOLOGİA CARDIOVASCULAR)}

\section{Antecedentes personales}

Se deben investigar los siguientes antecedentes, por la posibilidad de que indiquen la presencia de enfermedad cardiovascular:

- Enfermedad congénita o adquirida del sistema cardiovascular.

- Soplo cardiaco. Si se constata que se trata de un soplo funcional o inocente, no se considera un hallazgo patológico.

- Hipertensión arterial.

- Toma de medicación habitual actualmente o en los dos últimos años: considerando únicamente tratamientos farmacológicos prolongados, con el objetivo de identificar medicaciones con potenciales efectos adversos cardiovasculares.

- Crisis convulsivas no aclaradas: la importancia de este punto radica en que, en ocasiones, estos episodios se deben a eventos arrítmicos potencialmente letales. Las crisis epilépticas confirmadas no se incluyen en este apartado.

- Otras enfermedades: en último lugar se debe indagar sobre la presencia de cualquier enfermedad que el niño o su familia perciban como limitante para la práctica deportiva.

\section{Antecedentes familiares}

Hasta en un 40\% de los casos de MSC existe algún tipo de antecedente familiar (AF). Se deben investigar los siguientes AF:

- Cardiopatías congénitas (CC). Aunque solo el $6 \%$ de las causas de CC se deben a una anomalía cromosómica identificable, sabemos que el riesgo de tener CC aumenta cuando uno de los padres o hermanos son portadores de una CC.

- Muerte súbita antes de los 50 años: el 27\% de los casos de MSC refería AF de MSC en menor de 50 años.

- Enfermedades cardiovasculares en menores de 50 años: existen formas familiares de enfermedades cardiovasculares que pueden predisponer al desarrollo de MSC.

- Arritmias que hayan requerido tratamiento: los trastornos eléctricos suponen una de las causas más frecuentes de MSC, pero en la mayoría de los casos su penetrancia es incompleta y la expresión muy variable.

\section{Anamnesis}

La anamnesis debe estar dirigida a identificar los síntomas que pueden hacer sospechar la presencia de una enfermedad cardiovascular potencialmente letal.

- Dolor precordial: se buscarán las características del dolor sugestivo de isquemia coronaria (dolor opresivo, acompañado de sintomatología vegetativa y que interfiere inequívocamente con la actividad que se está realizando).

- Síncope o presíncope: recoger las circunstancias en que se produjo el episodio, especialmente si tuvo lugar durante el ejercicio físico o después del mismo.

- Palpitaciones: recoger los detalles del episodio, dado que la asociación de palpitaciones con otra sintomatología (mareo o síncope, dolor precordial, sudoración, nauseas, disnea) puede hacernos sospechar la presencia de cardiopatía.

- Disnea de esfuerzo: la disnea de esfuerzo puede ser el síntoma de presentación en algunas cardiopatías. 


\section{Exploración física}

Además de la exploración general completa, deberemos incidir en los siguientes puntos:

- Presión arterial (PA). Se debe incluir la toma de PA y determinación del percentil correspondiente $^{11}$. La PA normal se define como una PA sistólica y una PA diastólica inferiores al percentil 90, por grupo de edad, sexo y talla. Estos valores de referencia se han obtenido aplicando el método auscultatorio y, dado que los valores obtenidos en equipos oscilométricos son considerablemente mayores, los valores sugestivos de HTA por método oscilométrico deben confirmarse mediante método auscultatorio.

- Presencia de deformidades torácicas: pectus excavatum o carinatum. Algunas deformidades torácicas pueden afectar al sistema cardiovascular:

- En los pacientes afectos de pectus excavatum puede aparecer disminución del gasto cardiaco, prolapso mitral o arritmias. La disminución del gasto cardiaco es secundaria a la compresión sobre las cavidades cardiacas derechas por el esternón. Puede provocar menor tolerancia al ejercicio físico y taquicardia compensatoria. Se ha descrito prolapso mitral, relacionado con la compresión cardiaca. La prevalencia va aumentando con la edad. Es frecuente observar alteraciones electrocardiográficas en relación con la compresión y el desplazamiento cardiaco. Se han descrito bloqueo auriculoventricular de primer grado, bloqueo de rama derecha y síndrome de Wolff-Parkinson-White con mayor prevalencia que la población general, si bien la mayoría de las series no mencionan tal asociación.

- La bibliografía en relación a la afectación cardiovascular del pectus carinatum es muy escasa y variable. Se ha descrito en algunos trabajos una mayor prevalencia de anomalías valvulares mitrales (prolapso, insuficiencia o estenosis) y coartación de aorta, sin precisar porcentaje ni edad de aparición.
- Auscultación con valoración de soplos, tonos y ruidos sobreañadidos.

- Palpación de pulsos radiales y femorales.

- Presencia de estigmas de SM. El SM es una causa poco habitual de muerte súbita en el deportista joven. Se debe interrogar sobre la presencia de antecedentes familiares de SM y buscar estigmas de la enfermedad en la exploración física. En los niños y adultos jóvenes, los datos clínicos de afectación de la válvula mitral son más frecuentes que las lesiones aórticas. Pueden aparecer datos de insuficiencia mitral o prolapso mitral a la auscultación, siendo menos frecuente la detección de un soplo de insuficiencia aórtica.

Hay una serie de signos sistémicos que pueden detectarse en la exploración física y ante cuya presencia y asociación se debe valorar la remisión del paciente para estudio:

- Signo de la muñeca: la punta del pulgar cubre enteramente las uñas de los cinco dedos cuando envuelve la muñeca contralateral.

- Signo del pulgar: la falange distal del pulgar se extiende al borde cubital de la palma, con o sin ayuda del paciente o examinador para lograr la máxima abducción.

- Pectus carinatum o pectus excavatum.

- Deformidad retropié o pie plano.

- Relación segmento superior (talla-segmento inferior)/segmento inferior (distancia pubis al suelo estando de pie) reducida: ( $<1$ de cero a cinco años, $<0,95$ de seis a siete años, $<0,9$ de ocho a nueve años $y<0,85$ en mayores de diez años).

- Relación envergadura de brazos/estatura aumentada (>1,05).

- Escoliosis o cifosis toracolumbar.

- Extensión reducida del codo $\left(\leq 170^{\circ}\right.$ en extensión máxima).

- Hallazgos faciales (dolicocefalia, enoftalmos, fisura palpebral con inclinación inferior, hipoplasia malar, retrognatia).

- Estrías cutáneas.

- Miopía >3 dioptrías. 


\section{Pruebas complementarias}

EI ECG debe formar parte de la evaluación predeportiva de los niños y en la mayoría de los niños con síntomas durante el ejercicio. El ECG puede ser de gran utilidad para detectar:

- Miocardiopatías: miocardiopatía hipertrófica, displasia arritmogénica, miocardiopatía dilatada.

- Enfermedades del sistema de conducción: bloqueos auriculoventriculares, síndromes de preexcitación como el síndrome de Wolff-Parkinson-White.

- Enfermedades de los canales iónicos: síndromes de QT largo y corto, síndrome de Brugada.

\section{CASOS CLÍNICOS}

1. Una madre acude a la consulta con su hijo. Borja es un niño de 13 años de tu cupo, asmático bien controlado y sano por lo demás, que quiere jugar al baloncesto en el equipo el colegio. La madre está preocupada porque cuando Borja tenía 11 años le hicieron un ECG en Urgencias del hospital por un dolor de costado y le dijeron que tenía un "bloqueo de rama", aunque le explicaron entonces que no tenía ninguna importancia y que no necesitaba ningún seguimiento. También está preocupada porque en la familia padecen del corazón: la bisabuela paterna de Borja tenía el "corazón más grande que la caja" y el abuelo materno de Borja falleció a los 89 años de un infarto de miocardio. Decides explorarlo siendo la exploración normal salvo un soplo sistólico vibratorio I/VI en borde esternal derecho que desaparece con la sedestación. Realizas un ECG. Con la información del ECG, los antecedentes y la exploración debemos responder si Borja puede jugar sin problemas al baloncesto, si necesita hacerse alguna prueba más y si debe ser remitido a un servicio de cardiología infantil.

2. Susana es una niña de diez años que ayer por la tarde jugando en el patio del colegio "a pillar"y mientras corría en ambiente caluroso se empe- zó a marear, le dolió el pecho al respirar y se cayó al suelo golpeándose en el hombro y en la región frontal derechas. Un profesor que presenció lo ocurrido la cogió y la tumbó a la sombra, le levantaron las piernas y le echaron agua en la cara, Susana estaba muy pálida y al minuto abrió los ojos aunque tardó en hablar dos o tres minutos y estaba muy cansada. Al rato le dieron Coca-Cola ${ }^{\circledR}$ y a la media hora cuando llegó el padre estaba bien aunque pálida. Hoy acuden a la consulta porque aunque Susana tiene todas sus vacunas, la herida de la frente parece que se esta infectando y tiene un poco de arena que la madre no puede limpiar. En la consulta Susana tiene una PA 105/65, la auscultación y el resto de la exploración es normal. No hay antecedentes familiares de enfermedades del corazón. Susana tuvo un hermano previo fallecido a los dos meses de muerte súbita. Decides realizar un ECG.

3. Luis es un niño de 12 años. Hace un año, durante una consulta por un cuadro catarral sin fiebre, detectaste auscultación arrítmica; ya entonces realizaste un ECG que mostraba extrasístoles y lo enviaste a un servicio de cardiología infantil donde le hicieron un ecocardiograma y un holter siendo diagnosticado de "extrasistolia ventricular sin repercusión hemodinámica. No cardiopatía estructural", recomendando revisión en dos años. Hoy la madre trae a Luis porque le han empezado a picar los ojos como todas las primaveras. Cuando le auscultas percibes una arritmia marcada, como estás un rato auscultando cuando separas el fonendo la madre te mira a los ojos y con cara de preocupación pregunta: “¿Está todo bien?”. Tú le comentas que todo está bien, que tiene la arritmia de siempre pero que no tiene problema. La madre comenta que Luis juega al fútbol todos los días y pregunta si con esa arritmia puede hacer deporte. La revisión en Cardiología le corresponde dentro de un año. Piensas si tienes que adelantarle la cita por este motivo. Decides hacer un ECG.

4. Carlos es un adolescente de 16 años que acude a la consulta acompañado de sus padres porque 
esta mañana mientras juega un partido de fútbol presentó dolor torácico y sensación de falta de aire que le obligó a dejar el partido. Refiere que al parar se puso a sudar, le costaba respirar y tuvo sensación de estómago revuelto vomitando a los pocos minutos. Nunca había tenido antes palpitaciones, sincope ni dolor torácico. No hay antecedentes familiares de muerte súbita ni enfermedades cardiacas. A la exploración destaca un soplo sistólico eyectivo rudo III/VI en BEI. Se realiza un ECG cuyos hallazgos apoyan un diagnóstico que no se debe pasar.

5. Ana es una niña de 11 años traída a la consulta porque siempre que corre en la clase de gimnasia del colegio tiene dolor torácico tipo "pinchazo" no asociado a disnea, mareo, sudoración ni palidez, se para un poco (la madre dice que el profesor no les deja parar) y luego sigue corriendo sin problemas. Preguntada sobre la localización del dolor la niña se señala ambos hemitórax. No le pasa con otras actividades, va a baile y natación donde no han notado problemas. No hay antecedentes familiares de enfermedades cardiovasculares. La exploración física es normal. La madre te recuerda que es la segunda vez que trae a la niña por este motivo y pregunta si no habría que hacerle una prueba. Decides hacer un ECG que muestra un hallazgo no esperado.

6. Un adolescente de 16 años acude a la consulta con su madre por haber presentado un sincope ayer. Durante un partido de fútbol, tras jugar veinte minutos y descansar sentado en el banquillo a la sombra unos diez minutos, empezó a encontrarse mal con sensación de mareo y pérdida de conciencia con caída al suelo hacia delante desde sentado y golpeándose la frente. Se puso muy pálido, le tumbaron y recupero la conciencia casi de inmediato Había desayunado dos horas antes del episodio y estaba asintomático por lo demás. Su padre falleció de muerte súbita a los 41 años mientras dormía. Le exploras y decides hacer un ECG.

7. Andrés es un niño de 14 años que acude a un control rutinario de salud y a vacunarse. Du- rante la consulta, la madre comenta que Andrés juega al tenis y que este verano quiere apuntarse a un campamento intensivo de tenis. No hay antecedentes de enfermedad cardiovascular en la familia. La exploración muestra un pectus excavatum moderado y la auscultación del archivo sonoro que se reproducirá durante el taller. Con los hallazgos de la exploración debemos plantear una hipótesis diagnóstica y responder a la pregunta de la madre.

8. Carmen es una niña de ocho años traída a la consulta porque a los padres les parece que se cansa más que las compañeras. Viven en un primer piso sin ascensor y Carmen puede subir sin problemas, les parece que no podría subir un segundo o tercer piso sin parar. La niña no refiere sensación de corazón rápido, dolor torácico ni mareo. El profesor de gimnasia refiere que cuando corren Carmen se para más que las otras niñas aunque no sabe si es porque no le gusta correr. No hay antecedentes familiares de enfermedades cardiovasculares. A la auscultación, se detectan tonos ligeramente arrítmicos. Hacemos un ECG.

9. Teresa es una adolescente de 14 años que juega balonmano en el polideportivo de su pueblo. Entrena dos horas cuatro días en semana y juegan un partido los sábados. Esta federada y hace dos años, cuando se metió en el equipo le hicieron una revisión predeportiva con exploración física y un test de ejercicio con control de pulsaciones antes y después de hacer unas sentadillas que había pasado sin problemas. Acude con su padre porque a veces, cuando juega los partidos y en el entrenamiento, no sabe si es porque se pone nerviosa, nota que el corazón se le acelera no se acompaña de mareo ni dolor torácico pero como se pone un poco nerviosa a veces le cuesta respirar. Le preguntamos a Teresa una cuestión que nos orienta al diagnóstico. Realizamos un ECG que resulta muy clarificador.

10. Preguntas y casos de los asistentes. 


\section{CONFLICTO DE INTERESES}

El autor declara no presentar conflictos de intereses en relación con la preparación y publicación de este artículo.

\section{BIBLIOGRAFÍA}

1. DeBacker G, Ambrosioni E, Borch-Johnsen K, Brotons C, Cifkova R, Dallongeville J, et al. Third Joint Task Force of European and other societies on cardiovascular disease prevention in clinical practice: European Guidelines on cardiovascular disease prevention in clinical practice. Eur Heart J. 2003;24:1601-10.

2. Wren C. Screening children with a family history of sudden cardiac death. Heart. 2006;92:1001-6.

3. Corrado D, Migliore F, Basso C. Exercise and the risk of sudden cardiac death. Herz. 2006;31:553-8.

4. Firoozi S, Sharma S, McKenna WJ. Risk of competitive sport in young athletes with heart disease. Heart (British Cardiac Society). 2003;89:710-4.

5. Burke AP, Farb A, Virmani R. Sports-related and nonsports-related sudden cardiac death in young adults. Am Heart J. 1991;121:568-75.

6. Maron BJ, Doerer JJ, Haas TS. Profile and frequency of sudden death in 1463 young competitive athletes: from a 25 year US: national registry: 1980/2005. Circulation. 2006;114(Suppl II):830.

7. Maron BJ, Thompson PD, Ackerman MJ, Balady G, Berger S, Cohen D, et al.; American Heart Association Council on Nutrition, Physical Activity, and Metabolism. Recommendations and considerations related to preparticipation screening for cardiovascular abnormalities in competitive athletes: 2007 update: a scientific statement from the American Heart Association Council on Nutrition, Physical Activity, and Metabolism: endorsed by the American College of Cardiology Foundation. Circulation. 2007;115:1643-55.

\section{ABREVIATURAS}

AF: antecedente familiar - AHA: American Heart Association - CC: Cardiopatía congénita • ECG: electrocardiograma - ESC: Sociedad Europea de Cardiología • MSC: muerte súbita cardiaca • PA: presión arterial • SEC: Sociedad Española de Cardiología • SM: síndrome de Marfan.

8. Corrado D, Pelliccia A, Bjфrnstad HH, Vanhees L, Biffi A, Borjesson M, et al.; Study Group of Sport Cardiology of the Working Group of Cardiac Rehabilitation and Exercise Physiology and the Working Group of Myocardial and Pericardial Diseases of the European Society of Cardiology. Cardiovascular pre-participation screening of young competitive athletes for prevention of sudden death: proposal for a common European protocol. Consensus Statement of the Study Group of Sport Cardiology of the Working Group of Cardiac Rehabilitation and Exercise Physiology and the Working Group of Myocardial and Pericardial Diseases of the European Society of Cardiology. Eur Heart J. 2005;26:516-24.

9. Manonelles P, Aguilera B, Boraita A, Luengo E, Pons C, Suárez MP. Utilidad del electrocardiograma de reposo en la prevención de la muerte súbita del deportista. Documento de consenso de la Federación Española de Medicina del Deporte. Arch Med Deporte. 2007;24:159-68.

10. Boraita Pérez A, Baño Rodrigo A, Berrazueta Fernández JR, Lamiel Alcaine R, Luengo Fernández E, Manonelles Marqueta $P$, et al. Guías de práctica clínica de la Sociedad Española de Cardiología sobre la actividad física en el cardiópata. Rev Esp Cardiol. 2000;53:684-726.

11. National High Blood Pressure Education Program Working Group on High Blood Pressure in Children and Adolescents. American Academy of Pediatrics. The Second Report on the Diagnosis, Evaluation, and Treatment of High Blood Pressure in Children and Adolescents. Pediatrics. 2004;114:555-76. 